\title{
Measuring Research Impact of Astronomers/Astrophysicists by using Astrophysics Data System Beta: A Powerful New Interface: A case study with Special Reference to Prof. Jayant V. Narlikar
}

\author{
Hemant Kumar Sahu ${ }^{1, \star}$ and Surya Nath Singh ${ }^{2}$ \\ ${ }^{1}$ Inter-University Centre for Astronomy and Astrophysics, Ganeshkhind, Pune - 411007, India \\ ${ }^{2} \mathrm{Head} /$ Group leader, Information Centre \& Library, National Institute of Virology (ICMR), Pune - 411001, \\ India
}

\begin{abstract}
This paper highlights qualitatively and quantitatively research and presents a valuable overview of new citation enhanced databases in the context of research evaluation for the productivity of Prof. Jayant V. Narlikar. He has total 472 research publications in the fields of Astronomy and Astrophysics (AA) published from 1961-2015. In addition, this paper provides an overview of the citation-enhanced databases viz Astrophysics Data System Beta: A powerful new interface for performing citation analysis. Previously, scientometrics had been used to measure the publication productivity of Prof. Jayant V. Narlikar using the Astrophysics Data System (ADS). The scope of this paper is limited to Astrophysics Data System Beta. The result indicates that most of his papers are published in peer reviewed journals having the highest Impact Factor. The average number of publications per year is 8.74 and with the maximum papers published during 1981-1990 and 1996-2000. The total number of citations for his publications is 3516 covering 665 of his papers published during 1961-1970. He had many research collaborations, specifically with Prof. F. Hoyle (87 papers), Prof. G. Burbidge (38 papers), Prof. N. Wickramasinghe (22 papers) and Prof. T. Padmanabhan (21 papers).
\end{abstract}

\section{Introduction}

The great Indian Padmashree Prof. Jayant V. Narlikar is a well-known Astrophysicist and a proponent of Steady State Cosmology. He has developed the Hoyle-Narlikar theory of gravity with Sir F. Holye, which is a machian theory of gravity that fits into the quasi steady state model of the universe (Wikipedia, 2016). Recently he broke new grounds in space research. His research on space object is considered noteworthy. In addition to scientific publications, Prof. J.V. Narlikar is a writer of popular science articles, science fictions, short stories in English, Hindi and Marathi and also well known for communication of science through radio, and TV programs. He has been honored with many national and international awards. In this age of competition, quality and quantity have value. To value the publications, it is essential to measure the publication productivity of particular scientists. Scientometrics analysis is a useful method to collect information as well as an opportunity to access collections and services. Knowledge and survival are two sides of a coin in teaching and research.

\footnotetext{
^e-mail: hksahu@iucaa.in ORCID: 0000-0002-5588-8604
} 
Hence, the analysis and evolution of research is essential to find out its importance. There are various methods to measure the research in the form of articles, books, conference proceedings, and personal communications among the researchers. These are popularly known as Bibliometrics, Scientometrics Webometrics (also Cybermetrics), Informetrics, Productivity patterns, etc. It aims to estimate the varying contributions of scholarly work to the advancement of knowledge. The number of citations received by a publication is seen as a quantitative measure of the resonance and impact that this publication has produced in the scientific community [1]. The listings of references in publications are a convention among scientists for giving credit or recognition to the value of previous work [2]. There are commercial and free citation databases available via Web of Science, Scopus, Google Scholar and any subject-specific databases as such ADS: a Digital Library portal for researchers in astronomy and physics operated by the Smithsonian Astrophysical Observatory (SAO) under a NASA grant, now which also provide scientometrics of scientists. Web of Science is an online subscription-based scientific citation indexing service maintained by Thomson Reuters that provides a comprehensive citation search while Scopus is the largest abstracting and citation analysis database of peer-reviewed literature: scientific journals, books and conference proceedings. These citation databases are enriched with advanced features to enable detailed citation analysis along with h-index value. There is free citation databases such Google Scholar Citations, which provide a simple way for authors to keep track of citations to their articles and can check, who is citing his/her publications, graph citations over time, and compute several citation metrics and ADS is an online database of over twelve million astronomy and physics papers from both peer reviewed and non-peer reviewed sources. Astrophysics Data System Beta: A powerful new interface, which has chosen to explore out of the above said four databases being maximum used by IUCAA library users in their research, education requirement and citation analysis [3].

\subsection{Need of Study}

Publications are the main source of scientific communication. Research productivity in higher education and in research activities is gaining momentum and importance in the last ten years in India. It is the measurement of publication productivity that provides value to the research and denotes trends in that particular field. Citation analysis is used as an indicator of the impact valuing the quality and qualitative research. The work carried out by the Indian Scientists in the field of AA has international importance. Hence it is proper to measure/evaluate their publications to give the value at an international level. Researchers are always hungry for their new findings to give a new direction and contribute to their specific subject area, in forms of articles, patents, conference proceedings, books, chapter of books, etc. The result of such research has always been a source of further experiments and incalculable benefit to the society. Keeping this idea, the authors have made efforts to present this study with special reference to AA as a case study of Professor Jayant V. Narlikar, a globally well-known AA scientist, using of ADS beta version. There is no scientific research related to a study of the AA field in India. Hence, the present study is to find out some solutions for Library and Information Science (LISc) professionals as well as users. The major research questions are: Are we ready to explore citation analysis of scientists as individual and as group/collaborators via citation databases such as web of Science, SCOPUS, Google Scholar and ADS, etc.?, Are there problems by organizing/managing e-resources?, Are users of IUCAA aware of the capabilities (attitude, skill and knowledge) of accessing above citation databases?

\subsection{Objectives}

(i) To measure the publication productivity patterns of Prof. Jayant V. Narlikar in AA by using ADS beta: a powerful interface. (ii) To study and identify the contribution of Prof. Jayant V. Narlikar in 
the field of AA by measuring year wise, search result of refereed and non-refereed papers, citations, including self-citations, read and downloaded papers, author network, research collaborations, etc. (iii) To find out factors to motivate other scientists to write and publish their findings. (iv) To find out the authorship pattern of Prof. J. V. Narlikar. (v) To find out core collaborators of Prof. J.V. Narlikar in his research career.

\subsection{Scope and Limitations}

The IUCAA is a pioneer international institute for its teaching and research in the field of AA which includes main subjects: Astronomy, Astrophysics, Mathematics, Computer Science, Physics and its allied branches with more than 300 users comprising of senior faculty members, scientists, visiting associate, research scholars and non-teaching technical and supporting staff/trainees as well casual visitors and to facilitate them. Researchers have access to citation databases such as Web of Science and ADS database (free). Since it is a case study, scope and limitations are restricted to IUCAA and scientific publications of Prof. Jayant V Narlikar and scientometrics analysis as well as his aforesaid publications published during the period 1961-2015 [4].

\section{Review of Literature}

For the present study, many references were used such the ADS database and its related literature to explore citation analysis and get details of published papers/citations for the year. This is directly related to the present study covering AA subjects and its specialized users. The primary as well as the secondary sources of literature has been consulted. A number of studies have been conducted and no comprehensive and substantial studies have been carried out as a case study to understand and get citation analysis of an author via the ADS database. However, some study has been done by Christoph and Hans-Dietr, 2008, Stefanie, et al, 2014 and Helsinki University Main Library, 2016 but not directly related to a case study [5-9].

\section{Research Methods}

Scientific publications are the best source of scientific communications, while scientometrics is the best method to measure the contribution of individual scientists and/or growth of any discipline. To explore the details of any particular author's publications (Prof. Jayant V Narlikar) and citation patterns, the authors have used the scientometrics/citation analysis to evaluate and measure the research impact of astronomers/astrophysicist using an ADS Beta database. To explore the analysis, a case study of Prof. Jayant V. Narlikar has been taken and explored. For the purpose of the study, data was used from ADS database. A total of 472 publications have been analyzed. The bibliographic data of work carried out by Prof. J.V. Narlikar for the study were accessed from ADS. MS Office is used to organize tables and figures and analyses the data[4, 7, 9].

\section{Result, Discussion/Analysis and Major Findings}

\subsection{Author-wise Productivity and Citations}

Prof. J. V. Narlikar has published 472 papers during 1961-2015 (Table 1-2). The information about a data set was searched through ADS, for the papers published by Prof. Jayant V. Narlikar for the period of 54 years (From 1961 to 2015). The database was searched with necessary refinements to 
get information on published papers and citations in the ADS database which contains a maximum numbers of astronomy and astrophysics scholarly publications. ADS contain 472 papers published by Prof. Narlikar in scholarly international journals, books and conference proceedings with total numbers of 3516 citations [3].

\begin{tabular}{|r|l|l|r|}
\hline SN & $\begin{array}{l}\text { Five years du- } \\
\text { ration }\end{array}$ & $\begin{array}{l}\text { Numbers } \\
\text { of papers }\end{array}$ & Citations \\
\hline 1 & $1961-1965$ & 15 & 665 \\
\hline 2 & $1966-1970$ & 22 & 348 \\
\hline 3 & $1971-1975$ & 39 & 419 \\
\hline 4 & $1976-1980$ & 49 & 281 \\
\hline 5 & $1981-1990$ & 88 & 398 \\
\hline 6 & $1986-1990$ & 55 & 186 \\
\hline 7 & $1991-1995$ & 50 & 575 \\
\hline 8 & $1996-2000$ & 79 & 362 \\
\hline 9 & $2001-2005$ & 36 & 222 \\
\hline 10 & $2006-2010$ & 30 & 55 \\
\hline 11 & $2011-2015$ & 9 & 5 \\
\hline & Total & $\mathbf{4 7 2}$ & $\mathbf{3 5 1 6}$ \\
\hline
\end{tabular}

\begin{tabular}{|l|l|}
\hline Name of Co-Authors & $\begin{array}{l}\text { Nos of Publi- } \\
\text { cations }\end{array}$ \\
\hline Hoyle, F. & 87 \\
\hline Burbidge, G. & 38 \\
\hline Wickramasinghe, N. & 23 \\
\hline Padmanabhan, T. & 21 \\
\hline Chitre, S. & 17 \\
\hline Vishveshwara, C. & 14 \\
\hline Kembhavi, A. & 11 \\
\hline Packer, J. & 11 \\
\hline Arp, H. & 8 \\
\hline Vishwakarma, R. & 8 \\
\hline
\end{tabular}

Table-1 : Year wise (in Five Years Duration) Papers Published \& Citations Received (ADS, 2016)

Table-2 : Status of Research Collaborations (ADS, 2016)

Table-1 summarizes that Prof. Narlikar has published a total 472 papers, during 1961-2015 and an average of 8.7 papers per year with a total of 3516 citations with maximum papers published by him during the period 1981-1990. Also, a maximum numbers of citations (i.e. 665) for papers published during 1961 to 1965 for these papers. It can be concluded that period between 1981 1990 was the peak period for Prof. Narlikar from all points of view and maximum impact. Hence there is continued growth of publications. His total publications in the form of research output through different channels, includes journal articles $(64.40 \%)$, books $(07.62 \%)$, book chapters $(03.38 \%)$ and in the conference proceedings/reprints (14.40\%). His most preferred journals where Nature (35 papers), Bulletin of Astronomical Society of India (27 papers), Monthly Notices of the Royal Astronomical Society (21 papers) and so on.

After performing searches in the ADS modern form by Author surname "narlikar", the result is shown in table 1 that his total listed number of papers is 472 with the name of his research collaborators year wise during period 1961 - 2015. The maximum was during 1981-1985, while the minimum was during 2011-2015. It can be summarized that between 1961-1965 was beginning of his career while 2011-2015 has minimum research results. It also shows that there is a trend of growth during 1961 -1995, and it was increasing, while 1985 - 1990 and 2011-2015 was irregular rather than decreasing. Table 4 points out that Professor Jayant V. Narlkar has maximum research collaborations with Professor F. Hoyle (87 papers), Professor G. Burbidge (38 papers), Prof. N. Wickramasinghe (22 papers) and Prof. T. Padmanabhan (21 papers) (Table-2).

\subsection{Citation Metrics of Research papers by Prof. Jayant V. Narlikar}

Table $-1 \& 4$ shows that the author has total number of citations at 3516 as on September 15, 2016, a total number of self-citations at 641 , which is increasing day by day. It's also shown that the author 
has a total number of refereed citations - 3011 out of 3516 . It can be concluded that the citations by others are much more than the self-citations.

\begin{tabular}{|l|l|l|}
\hline Index & Total & Referred \\
\hline h-index & 33 & 30 \\
\hline m-index & 0.6 & 0.5 \\
\hline g-index & 50 & 45 \\
\hline i10-index & 89 & 82 \\
\hline i100-index & 4 & 3 \\
\hline tori-index & 94.5 & 70.5 \\
\hline riq-index & 176 & 152 \\
\hline Read10-index & 73.3 & 52.7 \\
\hline
\end{tabular}

\begin{tabular}{|l|l|l|}
\hline Publications & Total & Referred \\
\hline Numbers of citing papers & 2107 & 1724 \\
\hline Total citations & 3616 & 2965 \\
\hline Numbers of self-citations & 641 & 597 \\
\hline Average citations & 7.4 & 12.1 \\
\hline Median citations & 0 & 4 \\
\hline Normalized citations & 1849.2 & 1462.6 \\
\hline Referred citations & 3011 & 2616 \\
\hline Average refereed citations & 6.4 & 10.7 \\
\hline Median refereed citations & 0 & 3 \\
\hline Normalized refereed citations & 1567.4 & 1293.1 \\
\hline
\end{tabular}

Table- 3: Year-wis Status of Numbers of Indices of Author (ADS, 2016)
Table-4: Status of Numbers of Citations, Self- citations, etc and its Count Year-wise (ADS, 2016)

The aim of the above indices (Table-3) is to capture productivity as well as impact by counting how many of an author's papers have been cited, and by how many times; to have a h-index of 5 , five of a scholar's publications must have been cited by others at least five times each. While more sophisticated than plain citation counts, the h-index shares the limitations of in-comparability across fields and across career stages (Mcinearey Lab, 2016). It has been observed that there are a great variety of paper downloaded, cited and reads. The table 6 shows that the author hash -index count of 33, meaning his papers has been cited 33 times by others at least 33 times each.. It's also shows (table 8 ) the status of different indices such as m-index 0.6, g-index 50, i10-index 89, i100 index 4, tori index 176, risk index 176 and read 10-index is 67.1. It has been observed that via ADS Beta version database one can explore details of any particular author research impact such as its total citations, number of self-citations, average citations, median citations, normalized citations, refereed citations, average refereed citations, median refereed citations, normalized referred citations year wise, status of his research impact in types of indices such h-index, m-index, etc. year wise, status of numbers of reads, number of download with its count year wise, Status of groups of papers based on shared reference, status of frequently appearing and unique words in search result, status of result of read counts of author papers year wise. Prof. Narlikar, with his collaborators have published 472 papers during 1961-2015 in various areas of astronomy and astrophysics and allied subjects. Prof. Narlikar, with his collaborators have communicated their research findings and published their papers in mainly international journals of AA, which are indexed regularly in the ADS database and have a high impact factor. Prof. Narlikar has h-index count 33 that means his papers have been cited 33 times by others at least 33 times each.

\section{Conclusions}

It can be concluded that citation analysis of Prof. Narlikar has proved to be an important assessment tool for his research evaluation publication productivity, specifically the study of 54 years, during which time he had published 472 papers/8.7 per year. There are a number of co-authors with whom he did a major research collaboration during his scientific career. Many commercial and free citation databases are available such Web of Science, SCOPUS, Google Scholar, etc., But the ADS is free and 
powerful research tool and has had a significant impact on the efficiency of astronomical research since it was launched in 1992 as well as covering many old astronomical journals, proceedings, conferences. The use of ADS is almost universal among astronomers worldwide, and therefore ADS usage statistics as well as way to explore of citation analysis in unique, compare two available citation databases. The study suggests that citation-enhanced databases need to be examined carefully, with regard to both their potentialities and their limitations for citation analysis. Hence, on the above pattern, it is suggested to measure the research impact of other eminent scientists working on AA

\section{References}

[1] C. Neuhaus and D. Hans-Dieter, Data source for performing citation analysis: an overview in Journal of Documentation 64-2, 193-210 (2008)

[2] R.K. Merton, The Matthew Effect in Science, II: Cumulative Advantage and the Symbolism of Intellectual Property. ISIS. 79, 606-623 (1998). http://www.garfield.library.upenn.edu/merton/ matthewii.pdf

[3] Astrophysical Data System. (2016). http://ads.harvard.edu

[4] Inter-University Centre for Astronomy and Astrophysics (IUCAA). 2016). http://www.iucaa.in

[5] Helsinki University Main Library. 2016. Measuring research impact: a citation analysis tools. http://libraryguides.helsinki.fi/c.php?g=212738\&p=1403603

[6] Mach's principle. (2016). https://en.wikipedia.org/wiki/Mach\%27s_principle

[7] L. Meho, K. Yang, A new era in citation and bibliometric analysis: web of science, scopus and google scholar. (2006) https://arxiv.org/abs/cs/0612132v1

[8] Steady State Theoty. (2016). https://en.wikipedia.org/wiki/Steady_State_theoty

[9] S. Haustein, T.D. Bowman, K. Holmberg et al., Astrophysicists on Twitter: An in-depth analysis of tweeting and scientific publication behavior in Aslib Journal of Information Management 66-3, 279-296 (2014). https://doi.org/10.1108/AJIM-09-2013-0081

[10] Mcinearey Lab. (2016). http://mcinerneylab.com/research/h-index-m-index-and-google-citations 